\title{
On the exponent of the cokernel of the forget-control map on $\mathrm{K}_{0}$-groups
}

\author{
by \\ Francis X. Connolly (Notre Dame, IN) and \\ Stratos Prassidis (Buffalo, NY)
}

\begin{abstract}
For groups that satisfy the Isomorphism Conjecture in lower K-theory, we show that the cokernel of the forget-control $\mathrm{K}_{0}$-groups is composed by the $\mathrm{NK}_{0}$-groups of the finite subgroups. Using this information, we can calculate the exponent of each element in the cokernel in terms of the torsion of the group.
\end{abstract}

1. Introduction. The Farrell-Jones Isomorphism Conjecture states that certain geometrically significant spectra can be computed from the corresponding spectra of the virtually infinite subgroups of the fundamental group of the space. Such spectra are the K., L., A. and the pseudoisotopy spectrum.

In this paper we restrict our attention to groups that satisfy the Isomorphism Conjecture for lower K-groups. This class of groups includes many groups with interesting geometric properties ([6]).

Our approach is to define the controlled K-groups of a group as the part of the K-theory that can be detected by the K-theory of the finite subgroups. The controlled K-theory satisfies certain homological properties ([12], [1]). We will concentrate on the "other" part of the K-theory, i.e. the cokernel of the forgetful map from the controlled K-groups to K-groups.

TheOREm (Main Theorem). Let $\Gamma$ be a group that satisfies the Isomorphism Conjecture in lower K-theory. Then the cokernel of the forget-control map on $\mathrm{K}_{0}$-groups is generated by the $\mathrm{NK}_{0}$-groups of finite groups that are bases for the virtually infinite cyclic subgroups of $\Gamma$.

The method of the proof is based on the reduction of the problem to the computations of the cokernel of the forget-control map for virtually infinite cyclic groups. The assumption on $\Gamma$ implies that there is a Quinn-type

2000 Mathematics Subject Classification: 57N15, 19A31, 19J05, 19M05.

Research of S. Prassidis supported in part by a Research Grant from FAPERJ, Brazil. 
spectral sequence with $E^{2}$-term depending on the K-theory of the virtually infinite cyclic subgroups of $\Gamma$. On the other hand, a similar spectral sequence that depends on the K-theory of the finite subgroups of $\Gamma$ computes the controlled K-groups. The comparison of the two spectral sequences shows that the cokernel of the forgetful map on $\mathrm{K}_{0}$-groups can be computed from the cokernels of the forgetful maps on the K-groups of the virtually infinite cyclic subgroups. But such a cokernel consists of $\mathrm{NK}_{0}$-groups of the finite subgroups ([9]). Such a calculation allows us to determine the exponents of these elements using the results of [5].

The interest of the two authors in the cokernel of the forgetful map arises from problems in equivariant topology. It is known ([15], [14]) that the topological equivariant lower K-groups can be computed as the cokernel of the forgetful map. Computations of the exponent of such groups have applications in the study of equivariant rigidity problems ([4]). In [4] and [10], the cokernel of the forget-control map was connected to the equivariant topological K-groups. In both cases, the assumption on $\Gamma$ was that its finite quotient was a group of odd order. The result of the main theorem allows us to obtain information on the equivariant topological groups from the torsion of the group itself and not its quotient.

The second author would like to thank the Department of Mathematics at the University of Notre Dame for the hospitality in December 1999, when this work took its final form. Also, he would like to express his thanks to the Coordenação de Pós Graduação em Mathemática at Niterói, Brazil, where this work was developed.

2. Preliminaries. We will review the construction of homotopy colimits. A concise reference is [16]. Our approach will also use the definitions and calculations in [8]. All the functors will be covariant. A category $\mathcal{C}$ is called topological if the morphism sets are topological spaces and the composition maps are continuous. Notice that if $F: \mathcal{C} \rightarrow \mathcal{D}$ is a continuous functor between topological categories, then

$$
\mathcal{D}_{F}: \mathcal{C}^{\mathrm{op}} \times \mathcal{D} \rightarrow \text { Spaces }
$$

defined by $\mathcal{D}_{F}(c, d)=\operatorname{Mor}_{\mathcal{D}}(F(c), d)$ on objects (and in the obvious way on morphisms) is a continuous functor. Let $X: \mathcal{C} \rightarrow$ Spaces and $Y: \mathcal{C}^{\text {op }} \rightarrow$ Spaces be two continuous functors. A simplicial set $B(Y, \mathcal{C}, X)$ (called the bar construction) is defined in [8] such that the $n$-cells are

$$
\begin{aligned}
B_{n}(Y, \mathcal{C}, X)=\left\{\left(y ; f_{1}, \ldots, f_{n} ; x\right): f_{1} \circ \ldots \circ f_{n} \in \operatorname{Mor}_{\mathcal{C}}\left(c, c^{\prime}\right),\right. \\
\left.x \in X(c), y \in Y\left(c^{\prime}\right)\right\} .
\end{aligned}
$$

If $Y: \mathcal{C}^{\text {op }} \times \mathcal{D} \rightarrow$ Spaces, then $B(Y, \mathcal{C}, X)$ can be considered as a functor 


$$
\begin{aligned}
& B(Y, \mathcal{C}, X): \mathcal{D} \rightarrow \text { Spaces }, \\
& B_{n}(Y, \mathcal{C}, X)(d)=\left\{\left(y ; f_{1}, \ldots, f_{n} ; x\right): f_{1} \circ \ldots \circ f_{n} \in \operatorname{Mor}_{\mathcal{C}}\left(c, c^{\prime}\right),\right. \\
&\left.\quad x \in X(c), y \in Y\left(c^{\prime}, d\right)\right\} .
\end{aligned}
$$

Definition 2.1. Let $X: \mathcal{C} \rightarrow$ Spaces be a continuous functor. The homotopy colimit of $X$ is defined by

$$
\operatorname{hcolim}_{\mathcal{C}} X=B(*, \mathcal{C}, X) .
$$

REMARK 2.2. (1) Let Spectra be the category of spectra with morphisms strict maps of spectra ([1], [16]). If $X$ has values in the category of spectra then $\operatorname{hcolim}_{\mathcal{C}} X$ is a spectrum $([16])$. In this case the pointed version of the bar construction is used.

(2) More generally, if $Y: \mathcal{C}^{\mathrm{op}} \times \mathcal{D} \rightarrow$ Spaces and $X: \mathcal{C} \rightarrow$ Spectra, the pointed bar construction defines a functor $B(Y, \mathcal{C}, X): \mathcal{D} \rightarrow$ Spectra. The construction of [16], $\S 1.2$, can be carried out in this case.

(3) There are different definitions of the homotopy colimit in the literature. There is a comparison scheme given in [16].

Let $K$ be an abstract simplicial complex and $B=|K|$ its geometric realization. Let $p: E \rightarrow B$ be a stratified system of fibrations ([12], [6]) and $\mathcal{S}$. a spectrum-valued homotopy functor on the category of spaces. We denote by $\mathbf{H} .(B ; \mathcal{S} .(p))$ the Quinn homology spectrum associated to $p$ and by $H_{*}(B ; \mathcal{S} .(p))$ the homotopy groups of the spectrum. The main calculational tool for the homology for such homology groups is an Atiyah-Hirzebruch type spectral sequence:

$$
E_{i, j}^{2}=H_{i}\left(B ; \pi_{j} \mathcal{S} .(p)\right) \Rightarrow H_{i+j}(B, \mathcal{S} .(p)) .
$$

In many instances, we will be dealing with a special kind of stratified systems of fibrations. Let cat $(K)$ be the category associated to the simplicial complex $K$, i.e. the category with objects the simplices of $K$ and one morphism from $\sigma$ to $\tau$ if $\sigma$ is a face of $\tau$. It is classical that if $c: \operatorname{cat}(K)^{\mathrm{op}} \rightarrow$ Spaces is the constant functor with value a single point, then $\operatorname{hcolim}_{\text {cat }(K)^{\text {op }}} c=|K|$.

Definition 2.3. The map $p$ has a homotopy colimit structure if there is a functor $\mathbf{F}: \operatorname{cat}(K)^{\mathrm{op}} \rightarrow$ Spaces such that

(1) $E=\operatorname{hcolim} \mathbf{F}$.

(2) $p=\operatorname{hcolim} \nu$ where $\nu$ is the natural transformation from $\mathbf{F}$ to $c$.

REMARK 2.4. If the map $p$ has a homotopy colimit structure then the Quinn homology spectrum has a more recognizable definition ([1], [16], Theorem 2.2):

$$
\text { H. }(B ; \mathcal{S} .(p))=\operatorname{hcolim}_{\text {cat }(K)^{\text {op }}}(\mathcal{S} . \circ \mathbf{F}) .
$$

Let $X$ be any connected CW-complex with $\pi_{1}(X)=\pi$ and universal cover $\widetilde{X}$. We write $\mathcal{C}_{\pi}$ for the class of finite subgroups and $\mathcal{G}_{\pi}$ for the class 
of finite or virtually infinite cyclic subgroups of $\pi$, in other words the class $\mathcal{G}_{\pi}$ consists of all virtually cyclic subgroups of $\pi$. We denote by $E \mathcal{C}_{\pi}$ and $E \mathcal{G}_{\pi}$ the corresponding classifying spaces for the given class of subgroups ([6], Appendix). Write $B \mathcal{C}_{\pi}$, respectively $B \mathcal{G}_{\pi}$, for their orbit spaces under the action of $\pi$. The projection maps induce commutative diagrams:
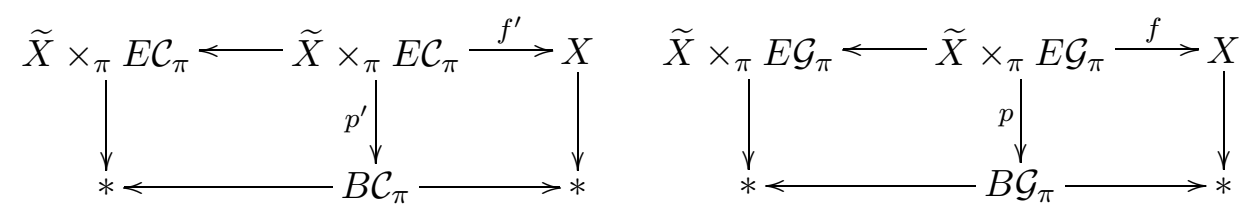

The left commutative squares induce maps, called assembly maps:

$$
\begin{aligned}
& A_{\mathcal{C}}: \mathbf{H} .\left(B \mathcal{C}_{\pi} ; \mathcal{S} .\left(p^{\prime}\right)\right) \rightarrow \mathcal{S} .\left(\tilde{X} \times_{\pi} E \mathcal{C}_{\pi}\right), \\
& A_{\mathcal{G}}: \mathbf{H} .\left(B \mathcal{G}_{\pi} ; \mathcal{S} .(p)\right) \rightarrow \mathcal{S} .\left(\widetilde{X} \times_{\pi} E \mathcal{G}_{\pi}\right)
\end{aligned}
$$

The Isomorphism Conjecture states that for $\mathcal{S}$. equal to the K-spectrum, $L$-spectrum, $A$-spectrum or pseudoisotopy spectrum, the composition

$$
\mathcal{S} .(f) \circ A_{\mathcal{G}}: \mathbf{H} .\left(B \mathcal{G}_{\pi} ; \mathcal{S} .(p)\right) \rightarrow \mathcal{S} .(X)
$$

is a homotopy equivalence. In [6], the Isomorphism Conjecture has been proved for a large class of groups for the pseudoisotopy spectrum. That implies that for the K-spectrum, the map $\mathcal{S}$. $(f) \circ A_{\mathcal{G}}$ induces an isomorphism on lower $(i \leq 1)$ homotopy groups.

Definition 2.5. We define the $\mathcal{S}$.-controlled spectrum of $X$ by

$$
\text { S. }(X)_{\mathrm{c}}=\mathbf{H} .\left(B \mathcal{C}_{\pi} ; \mathcal{S} \cdot\left(p^{\prime}\right)\right) .
$$

We call its homotopy groups the $\mathcal{S}$-controlled groups of the space $X$, i.e.

$$
S_{i}(X)_{\mathrm{c}}=H_{i}\left(B \mathcal{C}_{\pi} ; \mathcal{S} .\left(p^{\prime}\right)\right) .
$$

REMARK 2.6. (1) If $E \mathcal{C}_{\pi}$ and $E \mathcal{G}_{\pi}$ are given, then the product $E \mathcal{C}_{\pi} \times$ $E \mathcal{G}_{\pi}$, with the diagonal $\pi$-action, is a space of type $E \mathcal{C}_{\pi}$ ([6], Appendix).

(2) The universal properties of the classifying spaces for different classes of subgroups provide us with a unique map (up to $\pi$-homotopy) $u: E \mathcal{C}_{\pi}$ $\rightarrow E \mathcal{G}_{\pi}$. The map $u$ induces a commutative diagram

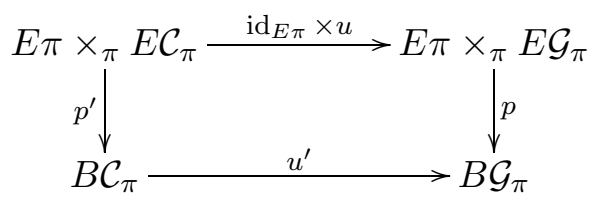

In Farrell-Jones ([6], Appendix) the map induced by $u$ on the Quinn homology spectra is denoted by $A_{\mathcal{C}, \mathcal{G}}$ and it is called the relative assembly map. 
Our main interest is in the case when $X=B \Gamma$ for some discrete group $\Gamma$. In this case, $\mathcal{S} .(f)$ is an isomorphism and the Isomorphism Conjecture can be reduced to showing that $A_{\mathcal{G}}$ is an isomorphism, or equivalently that the map induced by $f$ on the Quinn homology spectrum,

$$
\mathbf{H}(f): \mathbf{H} .\left(B \mathcal{G}_{\Gamma} ; \mathcal{S} .(p)\right) \rightarrow \mathcal{S} \text {. }(X),
$$

is a homotopy equivalence. We write $S_{i}(\Gamma)_{\mathrm{c}}$ for $S_{i}(X)_{\mathrm{c}}$ in this case. Also, the maps $p$ and $p^{\prime}$ have a homotopy colimit structure ([16], Lemma 4.1). The functor $\mathbf{F}^{\prime}: \operatorname{cat}\left(B \mathcal{C}_{\Gamma}\right)^{\text {op }} \rightarrow$ Spaces that induces the homotopy colimit structure is given by $\mathbf{F}^{\prime}(\tau)=\left(p^{\prime}\right)^{-1}(\widehat{\tau})$, the inverse image of the barycenter of the simplex. A similar construction applies to the space $B \mathcal{G}_{\Gamma}$. The functor $\mathbf{F}: \operatorname{cat}\left(B \mathcal{G}_{\Gamma}\right)^{\text {op }} \rightarrow$ Spaces is given by $\mathbf{F}(\sigma)=p^{-1}(\widehat{\sigma})$, as before. The details of the construction are given in [16].

REMARK 2.7. (1) We state the motivation of our definition for the controlled $S$-groups. Let $\Gamma$ be a virtually torsion-free group, i.e. there is an exact sequence

$$
1 \rightarrow \Gamma_{0} \rightarrow \Gamma \rightarrow G \rightarrow 1
$$

with $\Gamma_{0}$ torsion-free and $G$ finite. Let $M$ be a CW-complex of type $E \mathcal{C}_{\Gamma}$. Then the lower $(i \leq 1)$ reduced controlled K-group of $\Gamma$ defined above is isomorphic to the summand of $\widetilde{\mathrm{K}}_{i, G}^{\mathrm{PL}}\left(M / \Gamma_{0}\right)_{\mathrm{c}}$ corresponding to the pair $(M, \sigma(M))$ (here $\sigma(M)$ denotes the singular set of $M$ [4], [10], [14], [15]). The equivariant controlled PL-K-groups $\widetilde{\mathrm{K}}_{i, G}^{\mathrm{PL}}\left(M / \Gamma_{0}\right)_{\mathrm{c}}$ were introduced in [14] and [15] for the study of equivariant locally linear $h$-cobordisms. The equivariant controlled Whitehead group measures the failure of an equivariant PL-cobordism, which is topologically trivial, to be PL-trivial.

(2) There is a Quinn spectral sequence that computes the controlled S.-theory:

$$
E_{i, j}^{2}=H_{i}\left(B \mathcal{C}_{\Gamma} ; \pi_{j} \mathcal{S} \cdot\left(p^{\prime}\right)\right) \Rightarrow S_{i+j}(\Gamma)_{\mathrm{c}}
$$

and the coefficients depend only on the $\mathcal{S}$.-theory of the finite subgroups of $\Gamma$.

We are interested in the K-theory spectrum. We write

$$
\widetilde{\mathrm{K}}_{i}(\Gamma)= \begin{cases}\mathrm{K}_{i}(\mathbb{Z} \Gamma) & \text { for } i>1 \\ \mathrm{Wh}(\Gamma) & \text { for } i=1 \\ \widetilde{\mathrm{K}}_{0}(\mathbb{Z} \Gamma) & \text { for } i=0 \\ \mathrm{~K}_{i}(\mathbb{Z} \Gamma) & \text { for } i \leq 0\end{cases}
$$

Let $\widetilde{\mathbf{K}}$. be a functor from spaces to spectra such that

$$
\pi_{i}(\widetilde{\mathbf{K}} \cdot(X)) \cong \widetilde{\mathrm{K}}_{i}\left(\pi_{1}(X)\right) \quad \text { for } i \leq 1 .
$$

Such a functor exists ([11], [12]). 
Definition 2.8. A group $\Gamma$ is called a Farrell-Jones group for lower K-theory if the map determined by $f$,

$$
\mathbf{H} .\left(B \mathcal{G}_{\Gamma} ; \widetilde{\mathbf{K}} \cdot(p)\right) \stackrel{\mathbf{H}(f)}{\longrightarrow} \widetilde{\mathbf{K}} \cdot(B \Gamma),
$$

induces an isomorphism on $\pi_{i}$ for $i \leq 1$. For simplicity, we call such a group a Farrell-Jones group.

REMARK 2.9. (1) The general philosophy is that if a group has some reasonable geometric property then it is a Farrell-Jones group. Thus if a group $\Gamma$ is a discrete subgroup of isometries of $\mathbb{R}^{n}$ equipped with a Riemannian metric of non-positive curvature (with some mild extra conditions) then it is a Farrell-Jones group.

(2) Combining the definition with Quinn's spectral sequence we derive that, if $\Gamma$ satisfies the Isomorphism Conjecture, there is a spectral sequence

$$
E_{i, j}^{2}=H_{i}\left(B \mathcal{G}_{\Gamma} ; \pi_{j} \widetilde{\mathbf{K}} .(p)\right) \Rightarrow \widetilde{\mathrm{K}}_{i+j}(\Gamma) \quad \text { for } i+j \leq 1 .
$$

and the coefficients depend only on the K-theory of the virtually infinite cyclic subgroups of $\Gamma$.

3. Change of control. We will describe the controlled K-groups as Quinn homology groups over the space $B \mathcal{G}_{\Gamma}$. For this, we will use Quinn's "change of control" spectral sequence. But first we will give an explicit description of the Quinn homology groups using homotopy colimits (Remark 2.4).

Let $u^{\prime}: B \mathcal{C}_{\Gamma} \rightarrow B \mathcal{G}_{\Gamma}$ be the natural simplicial map. The map $u^{\prime}$ is well defined up to homotopy. So we fix a choice of $u^{\prime}$. Then $u^{\prime}$ induces a functor $U^{\prime}: \operatorname{cat}\left(B \mathcal{C}_{\Gamma}\right)^{\text {op }} \rightarrow \operatorname{cat}\left(B \mathcal{G}_{\Gamma}\right)^{\text {op }}$. Let $\mathbf{F}^{\prime}: \operatorname{cat}\left(B \mathcal{C}_{\Gamma}\right)^{\text {op }} \rightarrow$ Spaces, given by $\mathbf{F}^{\prime}(\tau)=\left(p^{\prime}\right)^{-1}(\widehat{\tau})$, be the functor that induces the homotopy colimit structure on $p^{\prime}([16], 4.1)$. For any simplex $\sigma$ in $\operatorname{cat}\left(B \mathcal{G}_{\Gamma}\right)^{\text {op }}$, we write $U^{\prime} \downarrow \sigma$ for the over-category of $\sigma$. In this case the category $U^{\prime} \downarrow \sigma$ can be identified with the subcategory of $\operatorname{cat}\left(B \mathcal{C}_{\Gamma}\right)^{\mathrm{op}}$ consisting of all the simplices $\tau$ such that $\sigma$ is a face of $u^{\prime}(\tau)$. Let $\mathbf{F}_{\sigma}^{\prime}: U^{\prime} \downarrow \sigma \rightarrow$ Spaces be the restriction $\mathbf{F}^{\prime} \mid\left(U^{\prime} \downarrow \sigma\right)$. Let

$$
h: \operatorname{cat}\left(B \mathcal{G}_{\Gamma}\right)^{\mathrm{op}} \rightarrow \text { Spaces, } \quad h(\sigma)=B\left(*, U^{\prime} \downarrow \sigma, \mathbf{F}_{\sigma}^{\prime}\right) .
$$

Notice that the image of $h$ is our model for the homotopy colimit, i.e. $h(\sigma)=$ hcolim $_{U^{\prime} \downarrow \sigma} \mathbf{F}_{\sigma}^{\prime}$. We will now establish a different representation of $h$.

LEMma 3.1. The following two functors are equal:

$$
B\left(\operatorname{cat}\left(B \mathcal{G}_{\Gamma}\right)_{U^{\prime}}^{\mathrm{op}}, \operatorname{cat}\left(B \mathcal{C}_{\Gamma}\right)^{\mathrm{op}}, \mathbf{F}^{\prime}\right)=h .
$$

Proof. We will just follow the definitions. The set of $n$-simplices of the first functor is given by 


$$
\begin{array}{r}
\left\{\left(y ; f_{1}, \ldots, f_{n} ; x\right): f_{n} \circ \ldots \circ f_{1} \in \operatorname{Mor}_{\mathrm{cat}\left(B \mathcal{C}_{\Gamma}\right)^{\mathrm{op}}\left(\tau, \tau^{\prime}\right), x \in \mathbf{F}^{\prime}(\tau),}\right. \\
y \in \operatorname{Mor}_{\left.\mathrm{cat}\left(B \mathcal{G}_{\Gamma}\right)^{\mathrm{op}}\left(U^{\prime}\left(\tau^{\prime}\right), \sigma\right)\right\} .}
\end{array}
$$

Because of the structure of the categories, the above set is equal to

$$
\left\{\left(* ; f_{1}, \ldots, f_{n} ; x\right): f_{n} \circ \ldots \circ f_{1}: \tau \rightarrow \tau^{\prime}, \tau^{\prime} \in U^{\prime} \downarrow \sigma, x \in \mathbf{F}^{\prime}(\tau)\right\} .
$$

But if $\tau^{\prime}$ belongs to the over-category $U^{\prime} \downarrow \sigma$ and there is a morphism from $\tau$ to $\tau^{\prime}$ in $\operatorname{cat}\left(B \mathcal{C}_{\Gamma}\right)^{\text {op }}$, then $\tau$ also belongs to $U^{\prime} \downarrow \sigma$. Thus the last set is exactly the set of $n$-simplices in $h(\sigma)$.

COROLlary 3.2. Let $\mathcal{S}$. be any homotopy functor from spaces to spectra. Then there is an equality of functors from the category cat $\left(B \mathcal{G}_{\Gamma}\right)^{\mathrm{op}}$ to spectra:

$$
B\left(\operatorname{cat}\left(B \mathcal{G}_{\Gamma}\right)_{U^{\prime}}^{\mathrm{op}}, \operatorname{cat}\left(B \mathcal{C}_{\Gamma}\right)^{\mathrm{op}}, \mathcal{S} . \circ \mathbf{F}^{\prime}\right)=\mathcal{S} . \circ h .
$$

The calculations done so far can be put together in describing the homotopy colimits that appear in our calculations of Quinn homology.

Proposition 3.3. The following two spectra are naturally equivalent:

$$
\operatorname{hcolim}_{\mathrm{cat}\left(B \mathcal{C}_{\Gamma}\right)^{\text {op }}}\left(\mathcal{S} . \circ \mathbf{F}^{\prime}\right) \cong \operatorname{hcolim} \operatorname{cat}_{\left(B \mathcal{G}_{\Gamma}\right)^{\text {op }}}(\mathcal{S} . \circ h) .
$$

Proof. The result follows from the more general result, the homotopy pushdown theorem for spectra $([8], 5.5)$. The theorem states that

$$
\begin{aligned}
\operatorname{hcolim}_{\operatorname{cat}\left(B \mathcal{C}_{\Gamma}\right)^{\mathrm{op}}}\left(\mathcal{S} . \circ \mathbf{F}^{\prime}\right) \\
\cong \operatorname{hcolim} \\
\qquad \operatorname{cat}\left(B \mathcal{G}_{\Gamma}\right)^{\text {op }} B\left(\operatorname{cat}\left(B \mathcal{G}_{\Gamma}\right)_{U^{\prime}}^{\text {op }}, \operatorname{cat}\left(B \mathcal{C}_{\Gamma}\right)^{\mathrm{op}}, \mathcal{S} . \circ \mathbf{F}^{\prime}\right) .
\end{aligned}
$$

The result follows from Corollary 3.2.

We will apply the previous results to our setting. We start by choosing an appropriate "change of control" map. The choice for $E \mathcal{G}_{\Gamma}$ used is the one given in the Appendix of [6]. It is the realization of a complex with $n$-simplex given by a sequence

$$
\sigma=g_{0} G_{0}\left(g_{1} G_{1}, g_{2} G_{2}, \ldots, g_{n} G_{n}\right)
$$

with $G_{i} \in \mathcal{G}_{\Gamma}$ such that $g_{i}^{-1} G_{i-1} g_{i} \subset G_{i}$ for $i=1, \ldots, n$. The face operator is given by

$$
\partial_{i} \sigma=\left\{\begin{array}{lr}
g_{0} g_{1} G_{1}\left(g_{2} G_{1}, \ldots, g_{n} G_{n}\right), & i=0, \\
g_{0} G_{0}\left(g_{1} G_{1}, \ldots, g_{i-1} G_{i-1}, g_{i} g_{i+1} G_{i+1}, g_{i+1} G_{i+1}, \ldots, g_{n} G_{n}\right), & 0<i<n, \\
g_{0} G_{0}\left(g_{1} G_{1}, \ldots, g_{n-1} G_{n-1}\right), & i=n .
\end{array}\right.
$$

The group $\Gamma$ acts on $E \mathcal{G}_{\Gamma}$ by

$$
\gamma \sigma=\left(\gamma g_{0}\right) G_{0}\left(g_{1} G_{1}, g_{2} G_{2}, \ldots, g_{n} G_{n}\right) \quad \text { for } \gamma \in \Gamma .
$$

The classifying space of the category $\operatorname{cat}\left(E \mathcal{G}_{\Gamma}\right)^{\mathrm{op}}$ is isomorphic as a simplicial set to the first derived subdivision $E \mathcal{G}_{\Gamma}^{\prime}$ of $E \mathcal{G}_{\Gamma}$. The group $\Gamma$ acts on $E \mathcal{G}_{\Gamma}^{\prime}$ by acting on each simplex in a chain, i.e. if $\sigma=\left[\sigma_{0}>\sigma_{1}>\ldots>\sigma_{n}\right]$ is 
a simplex in $E \mathcal{G}_{\Gamma}^{\prime}$ and $\gamma \in \Gamma$, then $\gamma \sigma=\left[\gamma \sigma_{0}>\gamma \sigma_{1}>\ldots>\gamma \sigma_{n}\right]$. Thus, for each simplex $\sigma$ of $E \mathcal{G}_{\Gamma}$ the classifying space of the over-category of the identity functor $\operatorname{Id} \downarrow \sigma$ is a subcomplex of the $E \mathcal{G}_{\Gamma}^{\prime}$. Let $\Gamma_{\sigma}$ be the isotropy group of $\sigma$. Then the action of $\Gamma$ induces an action of $\Gamma_{\sigma}$ on $\operatorname{Id} \downarrow \sigma$ and on its classifying space $|\operatorname{Id} \downarrow \sigma|$. In the next lemma we prove the equivariant properties of the last complex.

LEMMA 3.4. Let Id : $\operatorname{cat}\left(E \mathcal{G}_{\Gamma}\right)^{\mathrm{op}} \rightarrow \operatorname{cat}\left(E \mathcal{G}_{\Gamma}\right)^{\mathrm{op}}$ be the identity functor. Let $\sigma$ be a simplex of $E \mathcal{G}_{\Gamma}$ with isotropy group $\Gamma_{\sigma}$. Then the classifying space of the over-category $\operatorname{Id} \downarrow \sigma$, with the action induced by the action of $\Gamma_{\sigma}$ on the objects, is a space of type $E \mathcal{G}_{\Gamma_{\sigma}}$.

Proof. Let $\sigma=g_{0} G_{0}\left(g_{1} G_{1}, g_{2} G_{2}, \ldots, g_{n} G_{n}\right)$ with $\Gamma_{\sigma}=g_{0} G_{0} g_{0}^{-1}$. Let $\tau=g_{0}^{\prime} G_{0}^{\prime}\left(g_{1}^{\prime} G_{1}^{\prime}, g_{2}^{\prime} G_{2}^{\prime}, \ldots, g_{k}^{\prime} G_{k}^{\prime}\right)$ be an object in $\operatorname{Id} \downarrow \sigma$. Then $\tau$ contains $\sigma$ as a face. Thus $\Gamma_{\tau}$ is a subgroup of $\Gamma_{\sigma}$. If $\tau=\tau_{0}>\tau_{1}>\ldots>\tau_{n}$ is a simplex in $|\operatorname{Id} \downarrow \sigma|$ then $\Gamma_{\tau}=\Gamma_{\tau_{0}}$, which is a subgroup of $\Gamma_{\sigma}$. Thus all the isotropy groups of simplices in $\operatorname{Id} \downarrow \sigma$ belong to $\mathcal{G}_{\Gamma_{\sigma}}$. Let $H \in \Gamma_{\sigma}$. We will show that the fixed point set of the $H$-action on $|\operatorname{Id} \downarrow \sigma|$ is contractible. Actually, the idea presented in [6], p. 287, works in this case. In other words, the contraction defined there restricts to a contraction of the space $|\operatorname{Id} \downarrow \sigma|$. We give the details of the construction. Let $s$ be the contracting homotopy on $E \mathcal{G}_{\Gamma}^{H}$ that is constructed in [6], p. 287. More specifically, for a $k$-simplex $\tau=h_{0} H_{0}\left(h_{1} H_{1}, \ldots, h_{k} H_{k}\right)$ fixed by $H$, a $k+1$-simplex is constructed:

$$
s\left(h_{0} H_{0}\left(h_{1} H_{1}, \ldots, h_{k} H_{k}\right)\right)=H\left(h_{0} H_{0}, h_{1} H_{1}, \ldots, h_{k} H_{k}\right)
$$

with one zero face the 0 -simplex $H$. The construction $s$ defines the contraction to $H$. We construct the contraction of $|\mathrm{I} d \downarrow \sigma|^{H}$ in two steps. In the first step we define, for a $k$-simplex $\tau=\left[\tau_{0}>\tau_{1}>\ldots>\tau_{k}\right]$ in $|\operatorname{Id} \downarrow \sigma|$, a $k+1$-simplex

$$
S \tau=\left[s \tau_{0}>\tau_{0}>\tau_{1}>\ldots>\tau_{k}\right] .
$$

Then $\partial_{0} S \tau=\tau$ and $S$ commutes with the boundary maps. Thus $S$ defines a homotopy that contracts each simplex $\tau$ to $s \tau_{0}$. In the second step, notice that there is a 1 -simplex $\left[s \tau_{0}>s \sigma\right]$ from the last 0 -simplex to the 0 -simplex $s \sigma$ (notice that, since $H$ fixes $\sigma, s \sigma$ is a simplex in $\operatorname{Id} \downarrow \sigma$ ). The composition of the homotopies determined by the simplices above defines a contraction of $|\operatorname{Id} \downarrow \sigma|^{H}$ to the 0 -simplex $s \sigma$.

Let $E \mathcal{C}_{\Gamma}$ be chosen, for example by applying the same construction to the class $\mathcal{C}_{\Gamma}$. Then a different model for $E \mathcal{C}_{\Gamma}$ is the product $E \mathcal{C}_{\Gamma} \times E \mathcal{G}_{\Gamma}$ with the diagonal action of $\Gamma$. That is the model we will use in this section and we denote it by $E^{\prime} \mathcal{C}_{\Gamma}$. Let $u: E^{\prime} \mathcal{C}_{\Gamma} \rightarrow E \mathcal{G}_{\Gamma}$ be the projection map. Then $u$ induces a functor $U: \operatorname{cat}\left(E^{\prime} \mathcal{C}_{\Gamma}\right)^{\mathrm{op}} \rightarrow \operatorname{cat}\left(E \mathcal{G}_{\Gamma}\right)^{\mathrm{op}}$. The change of control map $u^{\prime}: B \mathcal{C}_{\Gamma} \rightarrow B \mathcal{G}_{\Gamma}$ is induced by the projection to the second space. The simplicial map $u^{\prime}$ induces a functor $U^{\prime}: \operatorname{cat}\left(B \mathcal{C}_{\Gamma}\right)^{\mathrm{op}} \rightarrow \operatorname{cat}\left(B \mathcal{G}_{\Gamma}\right)^{\mathrm{op}}$. Here the 
simplicial structure on the quotients is induced by the simplicial structure of the classifying spaces.

Let $[\sigma]$ be a cell in $B \mathcal{G}_{\Gamma}$, the orbit of a cell $\sigma$ of $E \mathcal{G}_{\Gamma}$. Then $[\sigma]$ is an object in $\operatorname{cat}\left(B \mathcal{G}_{\Gamma}\right)^{\text {op }}$. Let $U^{\prime} \downarrow[\sigma]$ be the over-category of $[\sigma]$ which, in this case, is equivalent to the subcategory of $\operatorname{cat}\left(B \mathcal{C}_{\Gamma}\right)^{\mathrm{op}}$ consisting of those simplices $[\tau]$ whose image, under $u^{\prime}$, contains $[\sigma]$. The next lemma gives a different characterization of the over-category.

Lemma 3.5. Let $\sigma$ be a simplex in $E \mathcal{G}_{\Gamma}$ with isotropy group $\Gamma_{\sigma}$.

(1) The geometric realization of the over-category $U \downarrow \sigma$, with the induced action, is a space of type ${E \mathcal{C}_{\Gamma_{\sigma}}}$.

(2) The geometric realization of $U^{\prime} \downarrow[\sigma]$ is a space of type $B \mathcal{C}_{\Gamma_{\sigma}}$.

Proof. For (1), notice that $U \downarrow \sigma$ can be described as the subcategory of $\operatorname{cat}\left(E^{\prime} \mathcal{C}_{\Gamma}\right)^{\text {op }}$ consisting of those simplices $\tau$ such that $u(\tau)$ contains $\sigma$. Here we identify the simplices with the objects of the corresponding categories. Since the map $u$ is the projection, $\tau=\tau^{\prime} \times \sigma^{\prime}$ with $\tau^{\prime}$ a simplex of $E \mathcal{C}_{\Gamma}$ and $\sigma^{\prime}$ a simplex of $E \mathcal{G}_{\Gamma}$ containing $\sigma$. The subcategory of all those simplices can be identified with $\operatorname{cat}\left(E \mathcal{C}_{\Gamma}\right)^{\mathrm{op}} \times(\operatorname{Id} \downarrow \sigma)$. Notice that $\Gamma_{\sigma}$ acts on the set of objects of the last category and it preserves the ordering. That induces an action on the geometric realization $E$ of the category. The complex $E$ is the product of the geometric realization $E \mathcal{C}_{\Gamma}$ of $\operatorname{cat}\left(E \mathcal{C}_{\Gamma}\right)^{\text {op }}$ and $E_{\sigma}$ of $\operatorname{Id} \downarrow \sigma$. The result follows from Lemma 3.4.

The second assertion follows from the relations

$$
\left|U^{\prime} \downarrow[\sigma]\right|=E \mathcal{C}_{\Gamma} \times \Gamma|U \downarrow \sigma| / \Gamma \stackrel{\cong}{\rightarrow} \mathcal{C}_{\Gamma} \times_{\Gamma_{\sigma}}|U \downarrow \sigma| .
$$

The first equality follows from the definitions. The homeomorphism is given by $[x, \gamma y] \mapsto[x, y]$.

THEOREM 3.6. There is a spectral sequence

$$
E_{i, j}^{2}=H_{i}^{\Gamma}\left(E \mathcal{G}_{\Gamma} ; \widetilde{\mathrm{K}}_{j}(G)_{\mathrm{c}}\right) \Rightarrow \widetilde{\mathrm{K}}_{i+j}(\Gamma)_{\mathrm{c}} \quad \text { for } i+j \leq 1
$$

where the $E^{2}$-term denotes Bredon homology and $G$ is in $\mathcal{G}_{\Gamma}$.

Proof. We use the result of Proposition 3.3. There are homotopy equivalences

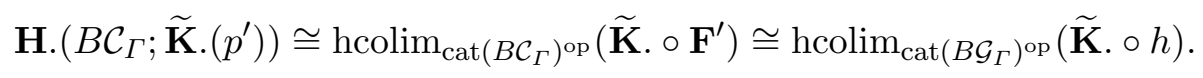

The functor $\mathbf{F}_{\sigma}^{\prime}$ induces a homotopy colimit structure for the map

$$
p_{\sigma}^{\prime}:\left(p^{\prime}\right)^{-1}\left(\left|U^{\prime} \downarrow[\sigma]\right|\right)=E \Gamma \times \Gamma_{\sigma}\left(E \mathcal{C}_{\Gamma} \times|U \downarrow \sigma|\right) \stackrel{p^{\prime} \mid}{\longrightarrow}\left|U^{\prime} \downarrow[\sigma]\right| .
$$

Thus, using the definitions, we obtain

$$
\begin{aligned}
\widetilde{\mathbf{K}} . \circ h([\sigma]) & =\operatorname{hcolim}_{U^{\prime} \downarrow[\sigma]}\left(\widetilde{\mathbf{K}} \cdot \circ \mathbf{F}_{\sigma}^{\prime}\right)=\mathbf{H} \cdot\left(\left|U^{\prime} \downarrow[\sigma]\right| ; \widetilde{\mathbf{K}} \cdot\left(p_{\sigma}^{\prime}\right)\right) \\
& =\mathbf{H} \cdot\left(B \mathcal{C}_{\Gamma_{\sigma}} ; \widetilde{\mathbf{K}} \cdot\left(p_{\sigma}^{\prime}\right)\right)=\widetilde{\mathbf{K}} \cdot\left(\Gamma_{\sigma}\right)_{\mathrm{c}}
\end{aligned}
$$


Taking homotopy groups we can have

$$
\pi_{j}(\widetilde{\mathbf{K}} . \circ h([\sigma]))=\widetilde{\mathbf{K}}_{j}\left(\Gamma_{\sigma}\right)_{c} .
$$

Now we can complete the argument as in [16]. Let $\mathcal{E}:$ AbGroups $\rightarrow$ Spectra be the Eilenberg-MacLane spectrum and $P$ : $\operatorname{cat}\left(E \mathcal{G}_{\Gamma}\right)^{\text {op }} \rightarrow \operatorname{cat}\left(B \mathcal{G}_{\Gamma}\right)^{\text {op }}$ be the functor induced by the quotient map $E \mathcal{G}_{\Gamma} \rightarrow B \mathcal{G}_{\Gamma}$. Let $\mathcal{O}(\Gamma)$ denote the orbit category of the group $\Gamma$ and $I: \operatorname{cat}\left(E \mathcal{G}_{\Gamma}\right)^{\mathrm{op}} \rightarrow \mathcal{O}(\Gamma)$ be the functor $I(\sigma)=\Gamma / \Gamma_{\sigma}$. We also define

$$
\begin{array}{ll}
M_{j}: \mathcal{O}(\Gamma) \rightarrow \text { AbGroups, } & M_{j}(\Gamma / H)=\widetilde{\mathrm{K}}_{j}(H)_{\mathrm{c}}, \\
M_{j}^{\prime}: \operatorname{cat}\left(B \mathcal{G}_{\Gamma}\right)^{\mathrm{op}} \rightarrow \text { AbGroups, } & M_{j}^{\prime}([\sigma])=\widetilde{\mathrm{K}}_{j}\left(\Gamma_{\sigma}\right)_{\mathrm{c}} .
\end{array}
$$

On morphisms the functors are defined using the induction maps ([4]). The functor $M_{*}^{\prime}$ is well defined because $\widetilde{\mathbf{K}}$. is a homotopy invariant functor ([16], Lemma 4.3). Also, by Lemma 4.4 of [16], there is a natural isomorphism $M_{j} I \cong M_{j}^{\prime} \circ P$. There is a spectral sequence

$$
E_{i+j}^{2}=H_{i}\left(B \mathcal{C}_{\Gamma} ; \pi_{j}\left(\widetilde{\mathbf{K}} \cdot\left(p^{\prime}\right)\right)\right) \Rightarrow \widetilde{\mathbf{K}}_{i+j}(\Gamma)_{\mathrm{c}} \quad \text { for } i+j \leq 1 .
$$

The $E^{2}$-term is defined as ( $\mathcal{E}$ denotes the Eilenberg-MacLane spectrum):

$$
\begin{aligned}
& H_{i}\left(B \mathcal{C}_{\Gamma} ; \pi_{j}\left(\widetilde{\mathbf{K}} \cdot\left(p^{\prime}\right)\right)\right) \\
& =\pi_{i}\left(\operatorname{hcolim}_{\mathrm{cat}}\left(B \mathcal{C}_{\Gamma}\right)^{\mathrm{op}}\left(\mathcal{E} \circ \pi_{j}(\widetilde{\mathbf{K}} \cdot) \circ \mathbf{F}^{\prime}\right)\right. \\
& =\pi_{i}\left(\operatorname{hcolim}_{\mathrm{cat}}\left(B \mathcal{G}_{\Gamma}\right)^{\mathrm{op}}\left(\mathcal{E} \circ \pi_{j}(\widetilde{\mathbf{K}} .) \circ h\right) \quad(\text { Proposition } 3.3)\right. \\
& =\pi_{i}\left(\operatorname{hcolim}_{\mathrm{cat}}\left(B \mathcal{G}_{\Gamma}\right)^{\mathrm{op}} \mathcal{E} M_{j}^{\prime}\right) \\
& \cong \pi_{i}\left(\operatorname{hcolim}_{\mathrm{cat}\left(E \mathcal{G}_{\Gamma}\right)^{\mathrm{op}}} \mathcal{E}\left(M_{j} I\right)\right) \\
& =H_{i}^{\Gamma}\left(E \mathcal{G}_{\Gamma} ; M_{j}\right)
\end{aligned}
$$

Because the isotropy groups of the simplices are in $\mathcal{G}_{\Gamma}$, the result follows.

For $\Gamma$ a Farrell-Jones group, there is a spectral sequence

$$
E_{i, j}^{2}=H_{i}\left(B \mathcal{G}_{\Gamma} ; \pi_{j} \widetilde{\mathbf{K}} .(p)\right) \Rightarrow \widetilde{\mathbf{K}}_{i+j}(\Gamma) \quad \text { for } i+j \leq 1 .
$$

The main result of [16] also implies that the $E^{2}$-term of the spectral sequence can be expressed as Bredon homology, i.e.

$$
E_{i, j}^{2}=H_{i}^{\Gamma}\left(E \mathcal{G}_{\Gamma} ; \widetilde{\mathrm{K}}_{j}(G)\right) \Rightarrow \widetilde{\mathrm{K}}_{i+j}(\Gamma) \quad \text { for } i+j \leq 1 .
$$

We would like to compare the last spectral sequence with the spectral sequence of Theorem 3.6.

Proposition 3.7. The forget-control map $\widetilde{\mathrm{K}}_{j}(G)_{\mathrm{c}} \rightarrow \widetilde{\mathrm{K}}_{j}(G)$ induces a map on the $E^{2}$-terms of the spectral sequences $(*)$ and $(* *)$. The latter map induces the forget-control map $\widetilde{\mathrm{K}}_{i+j}(\Gamma)_{\mathrm{c}} \rightarrow \widetilde{\mathrm{K}}_{i+j}(\Gamma)$ on the $E^{\infty}$-terms. 
Proof. We fix the notation. Let $[\sigma]$ be a simplex in $B \mathcal{G}_{\Gamma}$. Then we write

$$
\begin{aligned}
& p_{\sigma}^{\prime}:\left(p^{\prime}\right)^{-1}\left(U^{\prime} \downarrow[\sigma]\right)=E \Gamma \times_{\Gamma_{\sigma}}\left(E \mathcal{C}_{\Gamma} \times|U \downarrow \sigma|\right) \stackrel{p^{\prime} \mid}{\longrightarrow}\left|U^{\prime} \downarrow[\sigma]\right| \\
& p_{\sigma}: p^{-1}(\operatorname{Id} \downarrow[\sigma]) \quad=E \Gamma \times_{\Gamma_{\sigma}}|\operatorname{Id} \downarrow \sigma|
\end{aligned}
$$

Both maps have homotopy colimit structures. The functors are again given by taking the inverse images of the barycenters of simplices. Since $\Gamma_{\sigma}$ is a virtually infinite cyclic group and thus it is a Farrell-Jones group, we have isomorphisms

$$
\begin{aligned}
\widetilde{\mathrm{K}}_{j}\left(\Gamma_{\sigma}\right)_{\mathrm{c}} & =H_{j}\left(\left|U^{\prime} \downarrow[\sigma]\right| ; \widetilde{\mathbf{K}} .\left(p_{\sigma}^{\prime}\right)\right)=\pi_{j} \operatorname{hcolim}_{\operatorname{cat}\left(U^{\prime} \downarrow[\sigma]\right)} \widetilde{\mathbf{K}} .\left(\mathbf{F}^{\prime} \mid\right), \\
\widetilde{\mathrm{K}}_{j}\left(\Gamma_{\sigma}\right) & =H_{j}\left(|\operatorname{Id} \downarrow[\sigma]| ; \widetilde{\mathbf{K}} .\left(p_{\sigma}\right)\right)=\pi_{j} \operatorname{hcolim}_{\operatorname{cat}(\operatorname{Id} \downarrow[\sigma])} \widetilde{\mathbf{K}} .(\mathbf{F} \mid) .
\end{aligned}
$$

Also, the forget-control map is induced by the commutative diagram

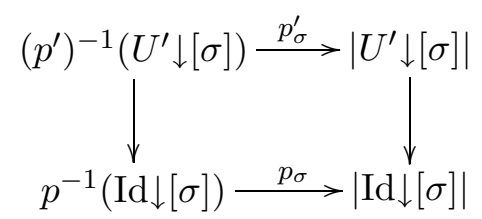

where the vertical maps are induced by the map $u$. For the spectral sequence $(*)$, the $E^{2}$-term is given as the homotopy groups of the spectrum

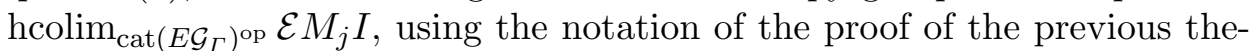
orem. Let

$$
\begin{array}{ll}
L_{j}: \mathcal{O}(\Gamma) \rightarrow \text { AbGroups, } & L_{j}(\Gamma / H)=\widetilde{\mathrm{K}}_{j}(H), \\
L_{j}^{\prime}: \operatorname{cat}\left(B \mathcal{G}_{\Gamma}\right)^{\mathrm{op}} \rightarrow \text { AbGroups, } & L_{j}^{\prime}([\sigma])=\widetilde{\mathrm{K}}_{j}\left(\Gamma_{\sigma}\right) .
\end{array}
$$

Also, let

$$
k: \operatorname{cat}\left(B \mathcal{G}_{\Gamma}\right)^{\text {op }} \rightarrow \text { Spaces, } \quad k([\sigma])=B(*, \operatorname{Id} \downarrow[\sigma], \mathbf{F} \mid(\operatorname{Id} \downarrow[\sigma])) .
$$

Then the sequence of isomorphisms in (1) can be carried out for the calculation of hcolim $\mathrm{cat}\left(E \mathcal{G}_{\Gamma}\right)^{\text {op }} \mathcal{E} L_{j} I$, whose homotopy groups are isomorphic to the $E^{2}$-term of the spectral sequence $(* *)$. We just have to replace the $M$-functors with the corresponding $L$-functors and the functor $h$ with $k$. At each stage of the process, the map $u$ induces a map between the corresponding spectra. Summarizing, there is a commutative diagram, up to homotopy:

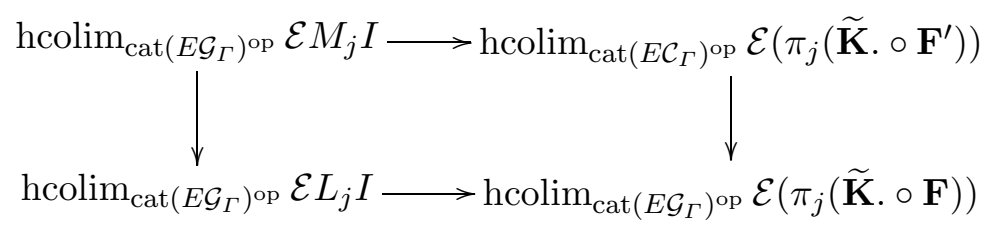

where the vertical maps are induced by $u$. Thus the map induced by the forget-control map on $E^{2}$-terms is the same as the map induced by the right 
vertical map in the diagram. But the last maps induces the forget-control map on the $E^{\infty}$-terms. The result follows.

We need more information about the forget-control map on virtually infinite cyclic groups. There are two types of virtually infinite cyclic groups:

- Type (1): Groups that admit an epimorphism onto the infinite cyclic group $\mathbb{Z}$ with finite kernel. Then the group $G$ has the form $G=H \rtimes_{\alpha} \mathbb{Z}$ with $H$ finite.

- Type (2): Groups that admit an epimorphism onto the infinite dihedral group $D_{\infty}$ with finite kernel. Then $G=G_{0} *_{H} G_{1}$ with $H$ finite and $\left[G_{0}: H\right]=\left[G_{1}: H\right]=2$.

Lemma 3.8. Let $G$ be a virtually infinite cyclic group. Then the forgetcontrol map

$$
\phi_{i}: \widetilde{\mathrm{K}}_{i}(G)_{\mathrm{c}} \rightarrow \widetilde{\mathrm{K}}_{i}(G) \quad \text { for } i \leq 1
$$

is a split monomorphism. Furthermore, the cokernel of $\phi_{i}$ is isomorphic to the corresponding Nil-groups.

Proof. For both types of virtually infinite cyclic groups, $E \mathcal{C}_{G}$ can be chosen to be $\mathbb{R}$ with $G$ acting through the projection to $\mathbb{Z}(\mathbb{Z}$ acts on $\mathbb{R}$ by translations $)$ in the first case and through $D_{\infty}\left(D_{\infty}\right.$ acts on $\mathbb{R}$ by translations and reflections) in the second. Then we get

$$
\begin{array}{ll}
E G \times_{G} \mathbb{R} \stackrel{p_{1}^{\prime}}{\longrightarrow} \mathbb{R} / G=\mathbb{R} / \mathbb{Z}=S^{1} & (\text { Type }(1)), \\
E G \times{ }_{G} \mathbb{R} \stackrel{p_{2}^{\prime}}{\longrightarrow} \mathbb{R} / G=\mathbb{R} / D_{\infty}=I & (\text { Type }(2)) .
\end{array}
$$

In those cases we can use the main result of [9] to get split exact sequences, for $i \leq 1$ :

$$
\begin{array}{lll}
0 \rightarrow \widetilde{\mathrm{K}}_{i}(G)_{\mathrm{c}} \rightarrow \widetilde{\mathrm{K}}_{i}(G) \rightarrow \mathrm{NK}_{i}(\mathbb{Z} H, \alpha) \oplus \mathrm{NK}_{i}\left(\mathbb{Z} H, \alpha^{-1}\right) \rightarrow 0 & \text { (Type (1)), } \\
0 \rightarrow \widetilde{\mathrm{K}}_{i}(G)_{\mathrm{c}} \rightarrow \widetilde{\mathrm{K}}_{i}(G) \rightarrow \mathrm{NK}_{i}\left(\mathbb{Z} H ; \mathbb{Z}\left[G_{0} \backslash H\right], \mathbb{Z}\left[G_{1} \backslash H\right]\right) \rightarrow 0 & \text { (Type (2)). }
\end{array}
$$

The result follows.

In [7], it was shown that $\widetilde{\mathrm{K}}_{i}(G)=0$ for $G$ a virtually cyclic group and for all $i \leq-2$. Using the Quinn spectral sequence that computes the controlled $\mathrm{K}$-groups, we see that $\widetilde{\mathrm{K}}_{i}(G)_{\mathrm{c}}=0$ for $i \leq-2$. Thus the spectral sequences $(*)$ and $(* *)$ collapse for $j \leq-2$. Computing the other K-groups of the virtually infinite cyclic groups involves the calculation of Nil-groups. In the first case, the Nil-groups appearing are the twisted classical Nil-groups, in the second case they are lower Waldhausen's Nil-groups.

Lemma 3.9. The Nil-groups appearing in the calculations of $\mathrm{K}_{-1}(G)$ for $G$ a virtually infinite cyclic group vanish. 
Proof. Type (1): Since $\mathrm{K}_{-2}(G)=0$, there is an exact sequence

$$
\mathrm{K}_{-1}(H) \stackrel{1-\alpha_{*}}{\longrightarrow} \mathrm{K}_{-1}(H) \stackrel{i_{*}}{\rightarrow} \mathrm{K}_{-1}(G) \rightarrow \mathrm{NK}_{-1}(\mathbb{Z} H, \alpha) \oplus \mathrm{NK}_{-1}\left(\mathbb{Z} H, \alpha^{-1}\right) \rightarrow 0 .
$$

In [7] it was shown that $\mathrm{K}_{-1}(G)$ is generated by the images of $\mathrm{K}_{-1}$ of its finite subgroups. But every finite subgroup of $G$ is isomorphic to a finite subgroup of $H$. Thus $i_{*}$ is an epimorphism, which implies that the $\mathrm{NK}_{-1^{-}}$ groups vanish.

Type (2): In this case, Waldhausen's exact sequence ([17], [18]) takes the form

$\mathrm{K}_{-1}\left(G_{0}\right) \oplus \mathrm{K}_{-1}\left(G_{1}\right) \stackrel{\left(i_{0},-i_{1}\right)}{\longrightarrow} \mathrm{K}_{-1}(G) \rightarrow \mathrm{NK}_{-1}\left(\mathbb{Z} H ; \mathbb{Z}\left[G_{0} \backslash H\right], \mathbb{Z}\left[G_{1} \backslash H\right]\right) \rightarrow 0$.

By the Corollary in [13], p. 36, every finite subgroup of $G$ is contained in a conjugate of $G_{0}$ or $G_{1}$. Since inner automorphisms induce the identity in K-theory, $\left(i_{0},-i_{1}\right)$ is an epimorphism, which implies that the $\mathrm{NK}_{-1}$-group vanishes.

LEMMA 3.10. For a virtually cyclic group $G$, the forget-control map $\mathrm{K}_{-1}(G)_{\mathrm{c}} \rightarrow \mathrm{K}_{-1}(G)$ is an isomorphism.

Proof. This follows from Lemmas 3.8 and 3.9.

Proposition 3.11. Let $\Gamma$ be a Farrell-Jones group. Then the forgetcontrol map $\mathrm{K}_{-1}(\Gamma)_{\mathrm{c}} \rightarrow \mathrm{K}_{-1}(\Gamma)$ is an isomorphism.

Proof. The result follows from the collapsing of the spectral sequences $(*)$ and $(* *)$ and Lemma 3.10:

$$
\mathrm{K}_{-1}(\Gamma)_{\mathrm{c}} \cong H_{0}\left(B \mathcal{G}_{\Gamma} ; \mathrm{K}_{-1}(G)_{\mathrm{c}}\right) \cong H_{0}\left(B \mathcal{G}_{\Gamma} ; \mathrm{K}_{-1}(G)\right) \cong \mathrm{K}_{-1}(\Gamma)
$$

Corollary 3.12. The map $H_{*}^{\Gamma}\left(E \mathcal{G}_{\Gamma} ; \mathrm{K}_{-1}(G)_{\mathrm{c}}\right) \rightarrow H_{*}^{\Gamma}\left(E \mathcal{G}_{\Gamma} ; \mathrm{K}_{-1}(G)\right)$ induced by the forget-control map is an isomorphism.

The next result is about the cokernel of the forget-control map on $\mathrm{NK}_{0^{-}}$ groups of virtually infinite cyclic groups. Let $G=H \rtimes_{\alpha} \mathbb{Z}$ or $G=G_{0} *_{H} G_{1}$. Let $|H|=n$ with $n=p_{1}^{k_{1}} \ldots p_{r}^{k_{r}}$ being the decomposition into primes. Let $l_{i}$, $i=1, \ldots, r$, be integers such that $p_{i}^{l_{i}} \geq k_{i} n$ for all $i$ and set $n^{\prime}=p_{1}^{l_{1}} \ldots p_{r}^{l_{r}}$.

Lemma 3.13. Let $G$ be a virtually infinite cyclic group. Then every element in the cokernel of the forget-control map $\phi_{0}: \widetilde{\mathrm{K}}_{0}(G)_{\mathrm{c}} \rightarrow \widetilde{\mathrm{K}}_{0}(G)$ has exponent $n^{\prime}$. In particular, the set of prime divisors of $n^{\prime}$ is equal to the set of prime divisors of $n$.

Proof. By Lemma 3.8, the cokernel of the forget-control map consists of $\mathrm{NK}_{0}$-groups. The result follows from [5].

4. The "forget-control" map on the $\widetilde{\mathrm{K}}_{0}$-groups. We are now in a position to state and prove the main result. 
TheOREM 4.1. The forget-control map $\phi: \widetilde{\mathrm{K}}_{0}(\Gamma)_{\mathrm{c}} \rightarrow \widetilde{\mathrm{K}}_{0}(\Gamma)$ is a monomorphism. Furthermore, the cokernel of $\phi$ is the epimorphic image of the direct sum of Nil-groups corresponding to the virtually infinite cyclic subgroups of $\Gamma$. Also, the set of primes that divide the exponent of an element in the cokernel of $\phi$ is the set of primes that divide the torsion of $\Gamma$.

Proof. Taking the edge exact sequences of the spectral sequences $(*)$ and $(* *)$ we obtain a commutative diagram with exact rows (we write $E \mathcal{G}$ and $B \mathcal{G}$ for $E \mathcal{G}_{\Gamma}$ and $B \mathcal{G}_{\Gamma}$, respectively):

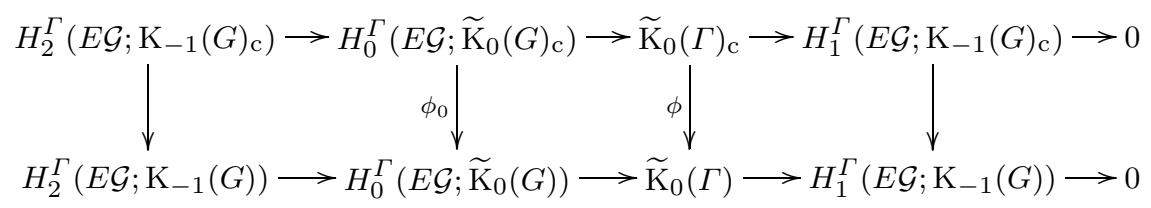

By Corollary 3.12, the first and the last vertical maps are isomorphisms. Also, the map $\phi_{0}$ is a split monomorphism. An easy diagram chase shows that $\phi$ is a monomorphism and that

$$
\operatorname{coker}\left(\phi_{0}\right)=\operatorname{coker}(\phi)
$$

Let $\Sigma$ be a complete set of orbit representatives of the action of $\Gamma$ on the zero skeleton of $E \mathcal{G}_{\Gamma}$. Then $H_{0}^{\Gamma}\left(E \mathcal{G} ; \widetilde{\mathrm{K}}_{0}(G)\right)$ is a quotient of the direct sum

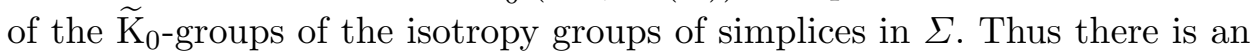
epimorphism

$$
\bigoplus_{\sigma \in \Sigma} \widetilde{\mathrm{K}}_{0}\left(\Gamma_{\sigma}\right) \rightarrow H_{0}^{\Gamma}\left(E \mathcal{G} ; \widetilde{\mathrm{K}}_{0}(G)\right)
$$

A similar result applies to the homology group $H_{0}^{\Gamma}\left(E \mathcal{G} ; \widetilde{\mathrm{K}}_{0}(G)_{\mathrm{c}}\right)$. Combining the above observations, we get a commutative diagram

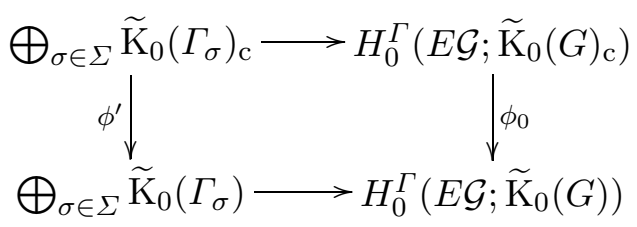

where the vertical maps are induced by the forget-control map. But the cokernel of $\phi^{\prime}$ is isomorphic to a direct sum of Nil-groups (Lemma 3.8). The result follows from Lemma 3.13 .

In [4] and [10], the cokernel of the forget-control map was connected to the equivariant topological K-groups. In both cases, the assumption on $\Gamma$ was that its finite quotient was a group of odd order. The assumption implies that $\Gamma$ does not contain virtually infinite cyclic subgroups of Type (2). Our result implies that we can replace the assumption on the finite quotient with an assumption on the group itself. To state the result, we assume that $\Gamma$ 
is a virtually torsion-free, discrete cocompact subgroup of isometries of a non-positively curved (in the sense of Gromov) topological manifold $\widetilde{M}$. Let

$$
1 \rightarrow \Gamma_{0} \rightarrow \Gamma \rightarrow G \rightarrow 1
$$

be an exact sequence such that $\Gamma_{0}$ is torsion-free and $G$ finite. We write $M=\widetilde{M} / \Gamma_{0}$ for the compact $G$-manifold induced by the action of $\Gamma_{0}$ on $\widetilde{M}$.

Proposition 4.2. Let $\Gamma$ be a group as above that does not have even torsion. With the above notation, $\mathrm{K}_{i, G}^{\mathrm{Top}}(M)=0$ for $i<0$, and $\widetilde{\mathrm{K}}_{0, G}^{\mathrm{Top}}(M)$ is a group with odd exponent. In particular,

$$
\widehat{H}^{*}\left(\mathbb{Z} / 2 \mathbb{Z} ; \widetilde{\mathrm{K}}_{0, G}^{\mathrm{Top}, \varrho}(M)\right)=0
$$

where $\mathbb{Z} / 2 \mathbb{Z}$ acts on $\widetilde{\mathrm{K}}_{0, G}^{\mathrm{Top}, \varrho}(M)$ by inverting the cobordisms.

Proof. In [14], there is an exact sequence connecting the different equivariant K-groups:

$$
\widetilde{\mathrm{K}}_{i, G}^{\mathrm{PL}}(M)_{\mathrm{c}} \rightarrow \widetilde{\mathrm{K}}_{i, G}^{\mathrm{PL}}(M) \rightarrow \widetilde{\mathrm{K}}_{i, G}^{\mathrm{Top}}(M) \rightarrow \widetilde{\mathrm{K}}_{i-1, G}^{\mathrm{PL}}(M)_{\mathrm{c}} \rightarrow \ldots, \quad i \leq 1 .
$$

In [14], the equivariant K-groups admit a splitting as a direct sum indexed by the components of the fixed point sets. The equivariant controlled groups split into a direct sum of controlled groups as defined in Definition 2.5 ([4]). The vanishing of the $\mathrm{K}_{i}$-groups, $i \leq-2$, follows from the vanishing of the lower K-groups of virtually infinite cyclic groups. That implies that the topological $\mathrm{K}_{-1}$-groups are isomorphic to the cokernel of the forget-control map. By Proposition 3.11, the forget-control map is an isomorphism, which implies the vanishing of the topological $\mathrm{K}_{-1}$-group. Similarly, the topological $\widetilde{\mathrm{K}}_{0}$-groups are given as the cokernel of the forget-control map. The first part of the proposition follows from 4.1. The last assertion follows from the fact that $\widetilde{\mathrm{K}}_{0, G}^{\text {Top, } \varrho}(M)$ is a summand of $\widetilde{\mathrm{K}}_{0, G}^{\text {Top }}(M)$ and from Theorem 4.1 .

\section{References}

[1] D. R. Anderson and H. J. Munkholm, Geometric modules and Quinn homology theory, K-Theory 7 (1993), 443-475.

[2] H. Bass, Algebraic K-Theory, Benjamin, 1968.

[3] D. W. Carter, Localization in lower K-theory, Comm. Algebra 8 (1980), 603-622.

[4] F. X. Connolly and T. Koźniewski, Rigidity and crystallographic groups I, Invent. Math. 99 (1990), 25-49.

[5] F. X. Connolly and S. Prassidis, On the exponent of the $\mathrm{NK}_{0}$-groups of virtually infinite cyclic groups, preprint.

[6] F. T. Farrell and L. E. Jones, Isomorphism conjectures in algebraic K-theory, J. Amer. Math. Soc. 6 (1993), 249-297.

[7] -, - , The lower algebraic $K$-theory of virtually infinite cyclic groups, $K$-Theory 9 (1995), 13-30. 
[8] J. Hollender and R. M. Vogt, Modules of topological spaces, applications to homotopy colimits and $E_{\infty}$ structures, Arch. Math. (Basel) 59 (1992), 115-129.

[9] H. J. Munkholm and S. Prassidis, Waldhausen's Nil-groups and continuously controlled K-theory, Fund. Math. 161 (1999), 217-224.

[10] S. Prassidis, A split exact sequence of equivariant $K$-groups of virtually nilpotent groups, K-Theory 11 (1997), 397-415.

[11] F. Quinn, Geometric algebra and ends of maps, lectures at the Univ. of Notre Dame.

[12] - Ends of maps II, Invent. Math. 45 (1982), 353-424.

[13] J. P. Serre, Trees, Springer, 1980.

[14] M. Steinberger, The equivariant h-cobordism theorem, Invent. Math. 45 (1988), 61-104.

[15] M. Steinberger and J. West, Equivariant h-cobordisms and finiteness obstructions, Bull. Amer. Math. Soc. 12 (1985), 217-220.

[16] R. Talbert, An isomorphism between Bredon and Quinn homology, Forum Math. 11 (1999), 591-616.

[17] F. Waldhausen, Whitehead groups of generalized free products, in: Algebraic $K$ Theory, II (Seattle, WA, 1972), Lecture Notes in Math. 342, Springer, 1973, 155-179.

[18] - Algebraic K-theory of generalized free products, Ann. of Math. 108 (1978), 135-256.

[19] M. Yamasaki, L-groups of virtually polycyclic groups, Topology Appl. 33 (1989), 223-233.

Department of Mathematics

University of Notre Dame

Notre Dame, IN 46556, U.S.A.

E-mail: connoly.1@nd.edu
Department of Mathematics Canisius College Buffalo, NY 14208, U.S.A. E-mail: prasside@canisius.edu

Received 7 June 2000;

in revised form 20 December 2001 